\title{
Correction to: Consensus guideline for the diagnosis and treatment of tetrahydrobiopterin (BH4) deficiencies
}

Thomas Opladen ${ }^{1 *}$, Eduardo López-Laso ${ }^{2+}$, Elisenda Cortès-Saladelafont ${ }^{3,4+}$, Toni S. Pearson ${ }^{5}, \mathrm{H}$. Serap Sivri ${ }^{6}$, Yilmaz Yildiz ${ }^{6}$, Birgit Assmann ${ }^{1}$, Manju A. Kurian ${ }^{7,8}$, Vincenzo Leuzzi ${ }^{9}$, Simon Heales ${ }^{10}$, Simon Pope ${ }^{10}$, Francesco Porta ${ }^{11}$, Angeles García-Cazorla ${ }^{3}$, Tomáš Honzík ${ }^{12}$, Roser Pons ${ }^{13}$, Luc Regal ${ }^{14}$, Helly Goez ${ }^{15}$, Rafael Artuch ${ }^{16}$, Georg F. Hoffmann ${ }^{1}$, Gabriella Horvath ${ }^{17}$, Beat Thöny ${ }^{18}$, Sabine Scholl-Bürgi ${ }^{19}$, Alberto Burlina ${ }^{20}$, Marcel M. Verbeek ${ }^{21}$, Mario Mastrangelo ${ }^{9}$, Jennifer Friedman ${ }^{22}$, Tessa Wassenberg ${ }^{14}$, Kathrin Jeltsch ${ }^{1 \dagger}$, Jan Kulhánek ${ }^{12^{*}{ }^{+}}$, Oya Kuseyri Hübschmann ${ }^{1 \dagger}$ and on behalf of the International Working Group on Neurotransmitter related Disorders (iNTD)

\section{Correction to: Orphanet Journal of Rare Diseases 15,} 126 (2020)

https://doi.org/10.1186/s13023-020-01379-8

Following the original article's publication [1] the authors asked for the correction of Fig. 2, since the names of the disease genes [GCH1 and PCBD1] in the figure published did not match the listed diseases [AR-GTPCHD and PCDD]. The correct Fig. 2 is shown below:

In the context of the manuscript correction and in order to match the text content, the words "apart from DHPRD" should be removed from the second row and second column of Table 4, as shown below:

The original article can be found online at https://doi.org/10.1186/s13023020-01379-8

* Correspondence: Thomas.Opladen@med.uni-heidelberg.de; Jan.Kulhanek@vfn.cz

†Thomas Opladen, Eduardo López-Laso, Elisenda Cortès-Saladelafont, Kathrin Jeltsch, Jan Kulhánek and Oya Kuseyri Hübschmann contributed equally to this work.

'Division of Child Neurology and Metabolic Disorders, University Children's

Hospital, Heidelberg, Germany

${ }^{12}$ Department of Paediatrics and Adolescent Medicine, First Faculty of

Medicine, Charles University and General University Hospital in Prague,

Prague, Czech Republic

Full list of author information is available at the end of the article

(c) The Author(s). 2020 Open Access This article is licensed under a Creative Commons Attribution 4.0 International License, which permits use, sharing, adaptation, distribution and reproduction in any medium or format, as long as you give appropriate credit to the original author(s) and the source, provide a link to the Creative Commons licence, and indicate if changes were made. The images or other third party material in this article are included in the article's Creative Commons licence, unless indicated otherwise in a credit line to the material. If material is not included in the article's Creative Commons licence and your intended use is not permitted by statutory regulation or exceeds the permitted use, you will need to obtain permission directly from the copyright holder. To view a copy of this licence, visit http://creativecommons.org/licenses/by/4.0/. The Creative Commons Public Domain Dedication waiver (http://creativecommons.org/publicdomain/zero/1.0/) applies to the data made available in this article, unless otherwise stated in a credit line to the data. 


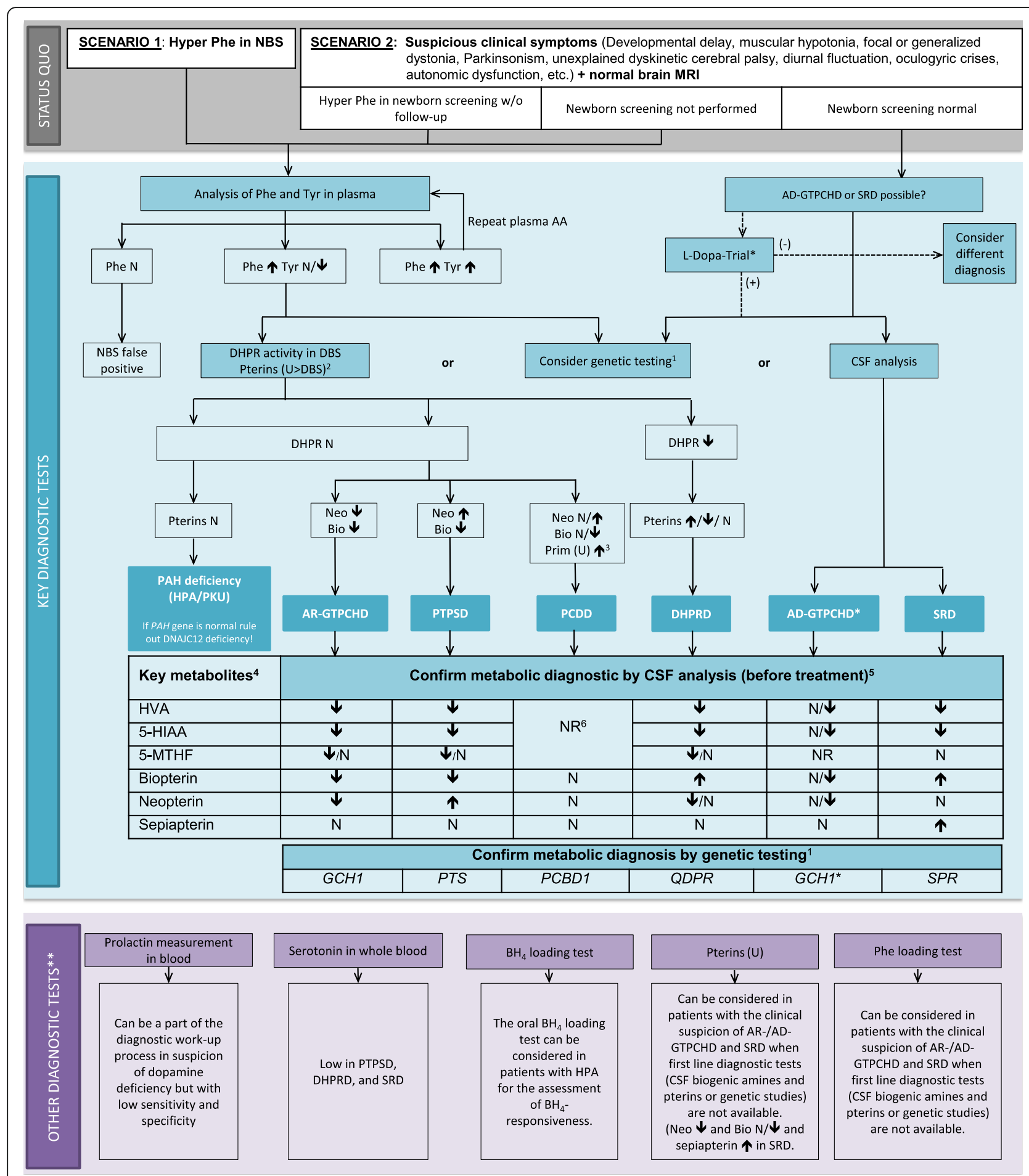

Fig. 2 (See legend on next page.) 
(See figure on previous page.)

Fig. 2 Diagnostic flowchart for differential diagnosis of $\mathrm{BH}_{4} \mathrm{Ds}$ with and without HPA. 'Consider genetic HPA workup depending on availability and financial resources. The gene panel should include the QDPR, GCH1, PTS PCBD1, SPR genes as well as DNAJC12. For GCH1, consider MLPA if Sanger sequencing is negative. ${ }^{2}$ The analysis in urine is more sensitive than in DBS and pathological patterns suggestive for PCDD and SRD can only be detected in urine but not in DBS. ${ }^{3}$ Primapterin measurement in urine is only elevated in PCDD. ${ }^{4}$ Aminoacids in CSF are not required for diagnosis of $\mathrm{BH}_{4} \mathrm{Ds} .{ }^{5} \mathrm{CSF}$ analysis should always include standard measurements (cell count, proteins, glucose and lactate). ${ }^{6}$ Recommendation against measurements of HVA, 5-HIAA, 5-MTHF, and pterins in CSF in the case of PCDD. $\left(^{*}\right)$ A diagnostic L-Dopa trial should be limited to children with symptoms suggestive of dopa-responsive dystonia or to situations where biochemical and genetic diagnostic tools are not available. If the diagnostic L-Dopa trial is positive but the results of CSF biochemical and/or molecular genetic testing are not compatible with AD-GTPCHD or SRD, further aetiologies for dopa responsive dystonia should be considered (e.g. juvenile parkinsonism (PARK2gene)). ${ }^{* *}$ ) Can be considered if available. See text for more detailed information. Abbreviations: 5-HIAA, 5-hydroxyindoleacetic acid; 5-MTHF, 5-methyltetrahydrofolate; AA: amino acids; AD-/AR- GTPCHD: guanosine triphosphate cyclohydrolase I deficiency; $\mathrm{BH}_{4}$, tetrahydrobiopterin; Bio: biopterin; CSF: cerebrospinal fluid; DBS: dry blood spot; DHPR: q-dihydropteridine reductase; DHPRD, dihydropteridine reductase deficiency; HVA, homovanillic acid; MRI, magnetic resonance imaging; N: normal; NBS: newborn screening; Neo: neopterin; NR: not reported; PAH: phenylalanine hydroxylase; Phe: phenylalanine; PKU: phenylketonuria; Prim: primapterin; PTPSD, 6-pyruvoyltetrahydropterin synthase deficiency; SRD: sepiapterin reductase deficiency; Tyr: tyrosine; u: urine; $(+)=$ positive effect; $(-)=$ no or no clear effect 
Table 4 Recommended drugs and doses for $\mathrm{BH}_{4}$ disorders

\begin{tabular}{|c|c|c|c|c|c|c|c|}
\hline & Disorder & Starting dose & Doses & Target dose & Maximum dose & $\begin{array}{l}\text { Management } \\
\text { suggestion }\end{array}$ & Comment \\
\hline \multicolumn{8}{|l|}{ First line treatments } \\
\hline Phe-reduced diet & $\begin{array}{l}\text { All } \mathrm{BH}_{4} \mathrm{D} \\
\text { with HPA }\end{array}$ & & & & & $\begin{array}{l}\text { Titrate Phe } \\
\text { restriction } \\
\text { according to } \\
\text { Phe levels in } \\
\text { DBS or plasma }\end{array}$ & $\begin{array}{l}\text { Follow PKU national treatment } \\
\text { recommendations } \\
\text { Use either Phe reduced diet or } \\
\text { Sapropterin dihydrochloride to } \\
\text { control Phe levels }\end{array}$ \\
\hline $\begin{array}{l}\text { Sapropterin } \\
\text { dihydrochloride }\end{array}$ & $\begin{array}{l}\text { All } \mathrm{BH}_{4} \mathrm{D} \\
\text { with } \mathrm{HPA}\end{array}$ & $\begin{array}{l}2-5 \mathrm{mg} / \mathrm{kg} \mathrm{BW/} \\
\text { day }\end{array}$ & $\begin{array}{l}\text { Divided } \\
\text { in } 1-3 \\
\text { doses/ } \\
\text { day }\end{array}$ & $\begin{array}{l}5-10 \mathrm{mg} / \mathrm{kg} \mathrm{BW/} \\
\text { day }\end{array}$ & 20 mg/kg BW/day & $\begin{array}{l}\text { Titrate dose } \\
\text { according to } \\
\text { Phe levels in } \\
\text { DBS or plasma }\end{array}$ & $\begin{array}{l}\text { Follow PKU national treatment } \\
\text { recommendations } \\
\text { Use either Phe reduced diet or } \\
\text { Sapropterin dihydrochloride to } \\
\text { control Phe levels }\end{array}$ \\
\hline $\begin{array}{l}\text { L-Dopa/DC inhibitor } \\
\text { (carbidopa/ } \\
\text { benserazide) } 4: 1\end{array}$ & $\begin{array}{l}\text { All } \mathrm{BH}_{4} \mathrm{D} \\
\text { apart from } \\
\text { PCDD }\end{array}$ & $\begin{array}{l}0.5 \mathrm{mg}-1 \mathrm{mg} / \mathrm{kg} \\
\text { BW/day } \\
\text { Dose } \\
\text { recommendation } \\
\text { relates to L-Dopa } \\
\text { component! }\end{array}$ & $\begin{array}{l}\text { Divided } \\
\text { in } 2-6 \\
\text { doses/ } \\
\text { day }\end{array}$ & $\begin{array}{l}\text { AD-GTPCHD: } \\
3-7 \mathrm{mg} / \mathrm{kg} \mathrm{BW/} \\
\text { day } \\
\text { All other } \mathrm{BH}_{4} \mathrm{D} \text { : } \\
10 \mathrm{mg} / \mathrm{kg} \mathrm{BW} \text { /day } \\
\text { or maximally } \\
\text { tolerated dosage } \\
\text { Dose } \\
\text { recommendation } \\
\text { relates to L-Dopa } \\
\text { component! }\end{array}$ & $\begin{array}{l}\text { Depending on } \\
\text { clinical symptoms. } \\
\text { Some patients need } \\
\text { more than } 10 \mathrm{mg} / \mathrm{kg} \\
\text { BW/day for resolving } \\
\text { clinical symptoms }\end{array}$ & $\begin{array}{l}\text { Increase } 0.5-1 \\
\text { mg/kg BW/day } \\
\text { per week } \\
\text { Follow BW } \\
\text { adaption until } \\
\text { the BW of } 40 \mathrm{~kg} \text {. } \\
\text { After } 40 \mathrm{~kg} \\
\text { adjust } \\
\text { depending on } \\
\text { clinical } \\
\text { symptoms } \\
\text { Consider } \\
\text { analysis of CSF } \\
\text { HVA for dose } \\
\text { adjustment }\end{array}$ & $\begin{array}{l}\text { In young infants at least as many } \\
\text { dosages as meals would be ideal } \\
\text { (usually 5-6/day) }\end{array}$ \\
\hline $\begin{array}{l}\text { 5- } \\
\text { Hydroxytryptophan } \\
\text { (5-HTP) }\end{array}$ & $\begin{array}{l}\text { All } \mathrm{BH}_{4} \mathrm{D} \\
\text { apart from } \\
\text { AD-GTPCHD } \\
\text { and PCDD }\end{array}$ & $\begin{array}{l}1-2 \mathrm{mg} / \mathrm{kg} \mathrm{BW/} \\
\text { day }\end{array}$ & $\begin{array}{l}\text { Divided } \\
\text { in 3-6 } \\
\text { doses/ } \\
\text { day }\end{array}$ & $\begin{array}{l}\text { Published target } \\
\text { dose } \\
\text { recommendations } \\
\text { are highly variable } \\
\text { 5-HTP doses are } \\
\text { usually lower than } \\
\text { L-Dopa doses }\end{array}$ & & $\begin{array}{l}\text { Titrate slowly } \\
\text { (1-2 mg/kg BW/ } \\
\text { day per week) } \\
\text { depending on } \\
\text { clinical picture } \\
\text { and side effects } \\
\text { Consider } \\
\text { analysis of CSF } \\
5 \text { HIAA for dose } \\
\text { finding }\end{array}$ & $\begin{array}{l}\text { 5-HTP should follow L-Dopa/DCI } \\
\text { treatment initiation } \\
\text { Always in combination with a } \\
\text { peripheral decarboxylase inhibitor } \\
\text { (for example by simultaneous } \\
\text { application with L-Dopa/DC } \\
\text { inhibitor) }\end{array}$ \\
\hline Folinic acid & $\begin{array}{l}\text { In DHPRD } \\
\text { and all } \\
\mathrm{BH}_{4} \mathrm{D} \text { with } \\
\text { low 5-MTHF } \\
\text { in CSF }\end{array}$ & & $\begin{array}{l}\text { Divided } \\
\text { in } 1-2 \\
\text { doses/ } \\
\text { day }\end{array}$ & 10-20 mg/day & & $\begin{array}{l}\text { No titration } \\
\text { needed } \\
\text { Consider } \\
\text { analysis of CSF } \\
5 \text { MTHF for dose } \\
\text { finding }\end{array}$ & \\
\hline \multicolumn{8}{|l|}{ Second line treatments } \\
\hline $\begin{array}{l}\text { Pramipexole }{ }^{\mathbf{a}} \\
\text { (Dopamine agonist) }\end{array}$ & $\begin{array}{l}\text { All } \mathrm{BH}_{4} \mathrm{D} \\
\text { apart from } \\
\text { PCDD }\end{array}$ & $\begin{array}{l}\text { 3.5-7 } \mu \mathrm{g} / \mathrm{kg} / \mathrm{BW} / \\
\text { day (base) } \\
5-10 \mu \mathrm{g} / \mathrm{kgBW} / \\
\text { day (salt) } \\
\text { Note: Distinction } \\
\text { in salt and base } \\
\text { content! } \\
\text { (see product } \\
\text { insert) }\end{array}$ & $\begin{array}{l}\text { Divided } \\
\text { in } 3 \\
\text { equal } \\
\text { doses/ } \\
\text { day }\end{array}$ & $\begin{array}{l}\text { Titrate to clinical } \\
\text { Symptoms }\end{array}$ & $\begin{array}{l}75 \mu \mathrm{g} / \mathrm{kg} \text { BW/day } \\
(3.3 \mathrm{mg} / \mathrm{d} \text { base } / 4 \\
\mathrm{mg} / \mathrm{d} \text { salt })\end{array}$ & $\begin{array}{l}\text { Increase } \\
\text { every } 7 \text { days by } \\
5 \mu \mathrm{\mu g} / \mathrm{kg} \mathrm{BW} / \mathrm{d}\end{array}$ & \\
\hline $\begin{array}{c}\text { Bromocriptine }{ }^{a} \\
\text { (Dopamine agonist) }\end{array}$ & $\begin{array}{l}\text { All } \mathrm{BH}_{4} \mathrm{D} \\
\text { apart from } \\
\text { PCDD }\end{array}$ & $\begin{array}{l}0.1 \mathrm{mg} / \mathrm{kg} \mathrm{BW/} \\
\text { day }\end{array}$ & $\begin{array}{l}\text { Divided } \\
\text { in } 2-3 \\
\text { doses/ } \\
\text { day }\end{array}$ & $\begin{array}{l}\text { Titrate to clinical } \\
\text { Symptoms }\end{array}$ & $\begin{array}{l}0.5 \mathrm{mg} / \mathrm{kg} / \mathrm{d} \\
\text { (or } 30 \mathrm{mg} / \mathrm{d} \text { ) }\end{array}$ & $\begin{array}{l}\text { Increase } \\
\text { every } 7 \text { days by } \\
0.1 \mathrm{mg} / \mathrm{kg} \mathrm{BW/d}\end{array}$ & \\
\hline $\begin{array}{l}\text { Rotigotine }^{\mathbf{a}} \\
\text { (transdermal dopamine } \\
\text { agonist) }\end{array}$ & $\begin{array}{l}\text { All } \mathrm{BH}_{4} \mathrm{D} \\
\text { apart from } \\
\text { PCDD }\end{array}$ & $2 \mathrm{mg} / \mathrm{day}$ & & $\begin{array}{l}\text { Titrate to clinical } \\
\text { Symptoms }\end{array}$ & $8 \mathrm{mg} / \mathrm{day}$ & $\begin{array}{l}\text { Increase weekly } \\
\text { by } \\
1 \mathrm{mg}\end{array}$ & $\begin{array}{l}\text { Children > } 12 \text { years } \\
\text { Exchange patch every } 24 \mathrm{~h}\end{array}$ \\
\hline $\begin{array}{l}\text { Selegiline }^{a} \\
\text { (MAO B inhibitor) }\end{array}$ & $\begin{array}{l}\text { All } \mathrm{BH}_{4} \mathrm{D} \\
\text { apart from } \\
\text { PCDD }\end{array}$ & $\begin{array}{l}0.1 \mathrm{mg} / \mathrm{kg} \mathrm{BW/} \\
\text { day }\end{array}$ & $\begin{array}{l}\text { Divided } \\
\text { in } 2 \\
(-3) \\
\text { doses/ } \\
\text { day }\end{array}$ & $\begin{array}{l}\text { Titrate to clinical } \\
\text { Symptoms }\end{array}$ & $\begin{array}{l}0.3 \mathrm{mg} / \mathrm{kg} / \mathrm{d} \\
(\text { or } 10 \mathrm{mg} / \mathrm{d} \text { ) }\end{array}$ & $\begin{array}{l}\text { Increase every } 2 \\
\text { weeks by } \\
0.1 \mathrm{mg} / \mathrm{kg} \text { BW/d }\end{array}$ & $\begin{array}{l}\text { Can cause sleep disturbances - } \\
\text { morning and afternoon or } \\
\text { lunchtime dosage is possible } \\
\text { ATTENTION: orally disintegrating } \\
\text { preparation needs much less } \\
\text { dosage because the first-pass ef- } \\
\text { fect of the liver is avoided }\end{array}$ \\
\hline
\end{tabular}


Table 4 Recommended drugs and doses for $\mathrm{BH}_{4}$ disorders (Continued)

\begin{tabular}{|c|c|c|c|c|c|c|c|}
\hline & Disorder & Starting dose & Doses & Target dose & Maximum dose & $\begin{array}{l}\text { Management } \\
\text { suggestion }\end{array}$ & Comment \\
\hline \multicolumn{8}{|l|}{ Third line treatments } \\
\hline $\begin{array}{c}\text { Trihexyphenidyl }^{\mathbf{a}} \\
\text { (Anticholinergic drugs) }\end{array}$ & $\begin{array}{l}\text { All } \mathrm{BH}_{4} \mathrm{D} \\
\text { apart from } \\
\text { PCDD }\end{array}$ & $\begin{array}{l}\text { < } 15 \text { kg: start } 0.5- \\
1 \text { mg/day } \\
>15 \mathrm{~kg}: \text { start } 2 \\
\text { mg/day }\end{array}$ & $\begin{array}{l}<15 \mathrm{~kg} \text { : } \\
\text { in } 1 \\
\text { dose } \\
>15 \mathrm{~kg}: \\
\text { in } 2 \\
\text { doses }\end{array}$ & $\begin{array}{l}\text { Effective dose } \\
\text { highly variable } \\
\text { (6-60 mg) } \\
\text { Titrate to clinical } \\
\text { Symptoms }\end{array}$ & $\begin{array}{l}\text { Maximum dose: } \\
<15 \mathrm{~kg} \mathrm{BW} \\
30 \mathrm{mg} / \mathrm{day} \\
>15 \mathrm{~kg} \mathrm{BW} \\
60 \mathrm{mg} / \mathrm{d}\end{array}$ & $\begin{array}{l}\text { Increase every } 7 \\
\text { days } \\
\text { by } 1-2 \mathrm{mg} / \mathrm{d} \text { in } \\
2-4 \text { doses/d }\end{array}$ & $\begin{array}{l}\text { Consider side effects: like dry } \\
\text { mouth, dry } \\
\text { eyes, blurred vision (mydriasis), } \\
\text { urine retention, constipation. }\end{array}$ \\
\hline $\begin{array}{l}\text { Entacapone }^{\mathbf{a}} \\
\text { (COMT inhibitor) }\end{array}$ & $\begin{array}{l}\text { All } \mathrm{BH}_{4} \mathrm{D} \\
\text { apart from } \\
\text { PCDD }\end{array}$ & $\begin{array}{l}200 \mathrm{mg} \\
\text { (adult) }\end{array}$ & & & Up to $2.000 \mathrm{mg}$ & & $\begin{array}{l}\text { In many countries licensed only } \\
\text { for adults. } \\
\text { Comedication with L-Dopa/DC } \\
\text { inhibitor } \\
\text { Consider reduction of } \\
\text { concomitant L-Dopa supplementa- } \\
\text { tion }(10-30 \%)\end{array}$ \\
\hline $\begin{array}{l}\text { Sertaline }^{\mathbf{a}} \\
\text { (SSRI) }\end{array}$ & $\begin{array}{l}\text { All } \mathrm{BH}_{4} \mathrm{D} \\
\text { apart from } \\
\text { PCDD }\end{array}$ & $\begin{array}{l}\text { 6-12 years: } 25 \\
\text { mg/day } \\
\text { in } 1 \text { dose } \\
>12 \text { years: } 50 \\
\text { mg/day in } 1 \text { dose }\end{array}$ & $\begin{array}{l}6-12 \\
\text { years: } \\
\text { in } 1 \\
\text { dose } \\
>12 \\
\text { years: } \\
\text { in } 1 \\
\text { dose }\end{array}$ & $\begin{array}{l}\text { Children } 50 \text { mg/ } \\
\text { day }\end{array}$ & $\begin{array}{l}50 \mathrm{mg} / \text { day } \\
<12 \text { years } \\
200 \mathrm{mg} / \text { day } \\
>12 \text { years }\end{array}$ & $\begin{array}{l}\text { 6-12 years: } \\
\text { increase after } 7 \\
\text { days to } 50 \mathrm{mg} / \\
\text { day } \\
\text { in } 1 \text { dose } \\
>12 \text { years } 50 \\
\text { mg/day in } 1 \\
\text { dose }\end{array}$ & $\begin{array}{l}\text { Don't stop treatment suddenly } \\
\text { Note: Elevated risk of serotonin } \\
\text { syndrome } \\
\text { (SS) or malignant neuroleptic } \\
\text { syndrome (MNS) when used with } \\
\text { drugs impacting serotonergic } \\
\text { pathway (e.g. 5-HTP, MAO } \\
\text { inhibitors) }\end{array}$ \\
\hline Melatonin $^{a}$ & $\begin{array}{l}\text { All } \mathrm{BH}_{4} \mathrm{D} \\
\text { apart from } \\
\text { PCDD }\end{array}$ & $\begin{array}{l}0.01-0.03 \mathrm{mg} / \mathrm{kg} / \\
\text { day }\end{array}$ & & & $5-8 \mathrm{mg} /$ day & & $\begin{array}{l}\text { Slow release preparation for sleep- } \\
\text { maintenance insomnia available in } \\
\text { some countries }\end{array}$ \\
\hline
\end{tabular}

Please note: The doses given are in a range typically used and have been published. In individual patients, some adjustment may be necessary depending on symptom response and side effects

${ }^{a}$ The evaluated literature did not provide $\mathrm{BH}_{4} \mathrm{D}$ specific treatment dose recommendations for this drug. The listed doses, therefore, indicate treatment recommendations from Summary of Product Characteristics (SmPC) or neurotransmitter related publications (e.g. [119])

Abbreviations: 5-HIAA 5-hydroxyindoleacetic acid, 5-HTP 5-hydroxytryptophan, 5-MTHF 5- methyltetrahydrofolate, HVA Homovanillic acid, AD-GTPCHD Autosomaldominant guanosine triphosphate cyclohydrolase I deficiency, $B H_{4} D$ Tetrahydrobiopterin deficiency, BW Body weight, COMT Catechol-O-methyl transferase, CSF Cerebrospinal fluid, DBS Dry blood spot, DC Decarboxylase, DCI Decarboxylase inhibitor, DHPRD Dihydropteridine reductase deficiency, L-Dopa L-3,4dihydroxyphenylalanine, MAO B Monoamine oxidase B, PCDD Pterin-4-alpha-carbinolamine dehydratase deficiency, Phe Phenylalanine, PKU Phenylketonuria, SSRI Selective serotonin reuptake inhibitor

\section{Author details}

'Division of Child Neurology and Metabolic Disorders, University Children's Hospital, Heidelberg, Germany. ${ }^{2}$ Pediatric Neurology Unit, Department of Pediatrics, University Hospital Reina Sofía, IMIBIC and CIBERER, Córdoba, Spain. ${ }^{3}$ Inborn errors of metabolism Unit, Institut de Recerca Sant Joan de Déu and CIBERER-ISCIII, Barcelona, Spain. ${ }^{4}$ Unit of Pediatric Neurology and Metabolic Disorders, Department of Pediatrics, Hospital Germans Trias i Pujol, and Faculty of Medicine, Universitat Autònoma de Barcelona, Badalona, Spain. ${ }^{5}$ Department of Neurology, Washington University School of Medicine, St. Louis, USA. ${ }^{6}$ Department of Pediatrics, Section of Metabolism, Hacettepe University, Faculty of Medicine, 06100 Ankara, Turkey. ${ }^{7}$ Developmental Neurosciences, UCL Great Ormond Street-Institute of Child Health, London, UK. ${ }^{8}$ Department of Neurology, Great Ormond Street Hospital, London, UK. 'Unit of Child Neurology and Psychiatry, Department of Human Neuroscience, Sapienza University of Rome, Rome, Italy. ${ }^{10}$ Neurometabolic Unit, National Hospital, Queen Square, London, UK. ${ }^{11}$ Department of Pediatrics, AOU Città della Salute e della Scienza, Torino, Italy. ${ }^{12}$ Department of Paediatrics and Adolescent Medicine, First Faculty of Medicine, Charles University and General University Hospital in Prague, Prague, Czech Republic. ${ }^{13}$ First Department of Pediatrics of the University of Athens, Aghia Sofia Hospital, Athens, Greece. ${ }^{14}$ Department of Pediatric, Pediatric Neurology and Metabolism Unit, UZ Brussel, Brussels, Belgium. ${ }^{15}$ Department of Pediatrics, University of Alberta Glenrose Rehabilitation Hospital, Edmonton, Canada. ${ }^{16}$ Clinical biochemistry department, Institut de Recerca Sant Joan de Déu, CIBERER and MetabERN Hospital Sant Joan de Déu, Barcelona, Spain. ${ }^{17}$ Department of Pediatrics, Division of Biochemical Genetics, BC Children's Hospital, University of British Columbia, Vancouver, BC, Canada. ${ }^{18}$ Division of Metabolism, University Children's Hospital Zurich, Zürich, Switzerland. ${ }^{19} \mathrm{Clinic}$ for Pediatrics I, Medical University of Innsbruck, Anichstr 35, Innsbruck, Austria. ${ }^{20}$ U.O.C. Malattie Metaboliche Ereditarie, Dipartimento della Salute della Donna e del Bambino, Azienda Ospedaliera Universitaria di Padova Campus Biomedico Pietro d'Abano, Padova, Italy. ${ }^{21}$ Departments of Neurology and Laboratory Medicine, Alzheimer Centre, Radboud University
Medical Center, Donders Institute for Brain, Cognition and Behaviour, Nijmegen, The Netherlands. ${ }^{22}$ UCSD Departments of Neuroscience and Pediatrics, Rady Children's Hospital Division of Neurology, Rady Children's Institute for Genomic Medicine, San Diego, USA.

Published online: 05 August 2020

\section{Reference}

1. Opladen, et al. Consensus guideline for the diagnosis and treatment of tetrahydrobiopterin (BH4) deficiencies. Orphanet Journal of Rare Diseases. 2020;15:126. https://doi.org/10.1186/s13023-020-01379-8. 